\title{
Performance of Wideband CDMA Using Adaptive Space-Time Spreading over Multipath Nakagami Fading Channels
}

\author{
Lie-Liang Yang and Lajos Hanzo \\ Dept. of ECS, University of Southampton, SO17 IBJ, UK. \\ Tel: +44-703-593 125, Fax: +44-703-594 508 \\ Email: ilyeecs.soton.ac.uk and Iheecs.soton.ac.uk \\ http://www-mobile.ecs.soton.ac.uk
}

\begin{abstract}
In this contribution an adaptive space-time spreading (ASTS) assisted transmit diversity scheme is proposed for improving the throughput of wideband code-division multiple-access (W-CDMA) systems. The performance of the system is investigated, when frequency-selective Nakagami- $m$ fading channels, multiuser interference and background noise are considered. Our numerical results demonstrate that the proposed ASTS-based transmission scheme is capable of significantly improving the effective throughpot of W-CDMA systems. Specifically, the studied W-CDMA system's bitrate can be increased by a factor of three at the modest cost of requiring an extra $0.4 \mathrm{~dB}$ or $1.2 \mathrm{~dB}$ transmitted power in the context of the investigated urban or suburban areas, respectively.
\end{abstract}

\section{INTRODUCTION}

The capacity and the achievabie data rate of wireless communication systems is limited by the time-varying characteristics of the channels. An efficient technique of combating the time-varying effects of wireless channels is employing diversity. In recent years, space-time coding has received much attention as an effective transmit diversity technique used for combating fading in wireless communications [1]. Inspired by space-time codes, in [2] an attractive transmit diversity scheme based on space-time spreading (STS) has been proposed for employment in CDMA systems. The performance of CDMA systems using STS has been investigated in $[2,3]$. The analysis and numerical results suggest that the achievable transmit diversity gain is independent of the frequency-selective diversity gain and that both the transmit diversity and the frequency-selective diversity have the same order of importance. Furthermore, the results recorded for transmission over frequency-selective fading channels show that when there is a sufficiently high number of resolvable paths generated by the specific environment encountered at the chip rate used, a CDMA system using a single transmit antenna and a conventional RAKE receiver is capable of achieving an adequate diversity gain.

This work has been funded in the framework of the IST project IST1999-12070 TRUST, which is partly funded by the European Union. The authors would like to acknowledge the contributions of their colleagues.

VTC'2002 Spring, Birmingham, Alabama, USA
The frequency-selective frequency-domain transfer function of W-CDMA wireless channels may vary slowly, but often over a wide dynamic range, when roaming in urban and suburban areas [5]. Therefore, the number of resolvable paths at the receiver can be modeled as a random variable distributed over a certain range, depending on the location of the receiver, where the number of resolvable paths varies slowly, as the receiver (mobile station) roams. Consequently, STS schemes designed on the basis of a low number of resolvable paths or based on the premise of encountering a constant number of resolvable paths may not achieve the maximum communication efficiency in terms of the effective throughput. Motivated by the above arguments, in this contribution an Adaptive STS (ASTS) based transmission scheme is proposed and investigated, which adapts the mode of operation of its STS scheme and its corresponding data rate, according to the near-instantaneous frequency-selectivity information fed back from the mobile receiver to the base station's transmitter. Our numerical results show that this adaptive STS scheme is capable of efficiently exploiting the diversity potential provided by the channel's frequency-selectivity, hence significantly improving the effective throughput of W.CDMA systems.

\section{SYSTEM MODEL}

\subsection{Transmitted Signal}

The W-CDMA system considered in this paper employs $U$ transmitter antennas and one receiver antenna. The transmitter schematic of the $k$ th user and the receiver schematic of the reference user are shown in Fig.1, where real-valued data symbols using BPSK modulation and real-valued spreading [2] were assumed. As shown in Fig.1(a), at the transmitter side the binary input data stream having a bit duration of $T_{b}$ is serial-to-parallel (S/P) converted to $U$ parallel sub-streams. The new bit duration of each parallel sub-stream, in other words the symbol duration, becomes $T_{s}=U T_{b}$. After $\mathrm{S} / \mathrm{P}$ conversion, the $U$ number of parallel bits are direct-sequence spread using the STS schemes proposed in [2] with the aid of $U$ number of orthogonal spreading sequences - for example Walsh codes - having a period of $U G$, where $G=T_{b} / T_{c}$ represents the number of chips per bit and $T_{\mathrm{c}}$ is the chip-duration of the orthogonal spreading sequences. As seen in Fig.1(a), following STS, the $U$ parallel signals to be mapped to the $U$ transmission antennas are scrambled using the $k$ th user's pseudo-noise (PN) sequence $\mathrm{PN}_{k}(t)$, in order that the transmitted signals become randomized, and to ensure that 
the orthogonal spreading sequences employed within the STS block of Fig. 1 can be reused by the other mobiles. Finally, after the PN sequence based scrambling, the $U$ number of parallel signals are carrier modulated and transmitted by the corresponding $U$ number of antennas. In this contribution we assume that the number of parallel data sub-streams, the number of orthogonal spreading sequences used by the STS block of Fig.I and the number of transmission antennas is the same, namely $U$. As shown in [2] this specific STS scheme constitutes an attractive scheme, since it is capable of providing maximal transmit diversity without requiring extra STS codes.
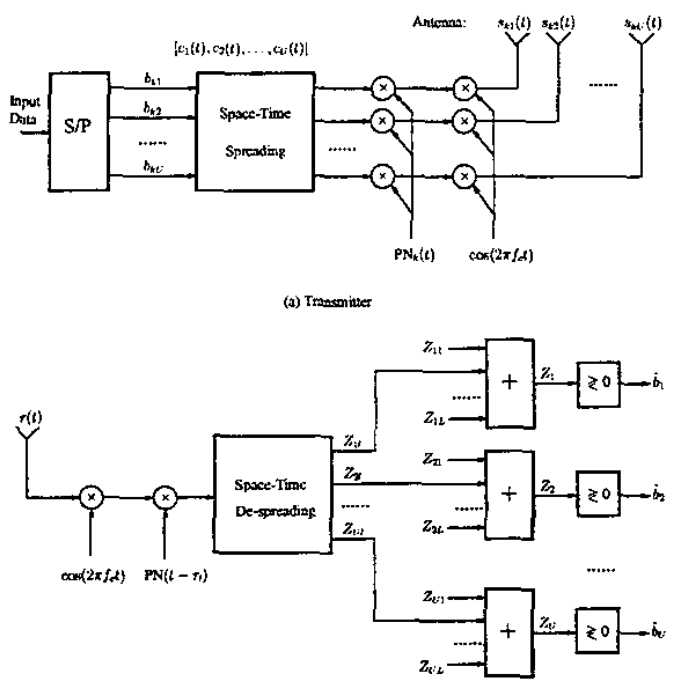

(b) Roxivive

Figure 1: Transmitter and receiver block diagram of the W-CDMA system using space-time spreading.

Based on the philosophy of STS as discussed in $[2,3]$ and refening to Fig.1(a), the transmitted signal of the $k$ th user can be expressed as

$$
\mathbf{s}_{k}(t)=\sqrt{\frac{2 P}{U^{2}}} \mathbf{c}(t) \mathbf{B}_{U}(t) \times \mathrm{PN}_{k}(t) \cos \left(2 \pi f_{\mathrm{c}} t\right),
$$

where $P$ represents each user's transmitted power, which is constant for all users. Furthermore, $s_{k}(t)=\left[s_{k 1}(t) s_{k 2}(t) \ldots s_{k U}(t)\right]$ represents the transmitted signal vector of the $U$ transmission antennas, while $\mathrm{PN}_{k}(t)$ and $f_{c}$ represent the DS scrambling based spreading waveform and the subcarrier frequency, respectively. The scrambling sequence waveform is given by $\mathrm{PN}_{k}(t)=\sum_{j=-\infty}^{\infty} p_{k j} P_{T_{c}}(t-$ $\left.j T_{c}\right)$, where $p_{k j}$ assumes values of +1 or -1 with equal probability, while $P_{T_{\mathrm{c}}}(t)$ is the rectangular chip waveform, which is defined over the interval $\left[0, T_{c}\right) . \operatorname{In}(1)$ the vector $\mathbf{c}(t)=\left[c_{1}(t) c_{2}(t) \ldots c v(t)\right]$ is constituted by the $U$ number of orthogonal signals assigned for the STS. Furthermore, $c_{i}(t)=\sum_{j=-\infty}^{\infty} c_{i j} P_{T_{c}}\left(t-j T_{c}\right), i=$ $1,2, \ldots, U$ denotes the individual components of the STS-based orthogonal spread signals, where $\left\{c_{i j}\right\}$ is an orthogonal sequence of period $U G$ for each index $i ; \mathbf{B}_{U}(t)$ represents the $U \times U$ dimensional transmitted data matrix created by mapping $U$ input data bits to the $U$ parallel sub-streams according to specific design rules [2] so that the maximum possible grade of transmit diversity is achieved, while using relatively low-complexity signal detection algorithms. Specifically, $\mathbf{B}_{U}(t)$ can be expressed as

$\mathbf{B}_{U}(t)=\left(\begin{array}{cccc}a_{11} b_{k, 11} & a_{12} b_{k, 12} & \ldots & a_{1 U} b_{k, 1 U} \\ a_{21} b_{k, 21} & a_{22} b_{k, 22} & \ldots & a_{2 U} b_{k, 2 U} \\ \vdots & \vdots & \ddots & \vdots \\ a_{U 1} b_{k, U 1} & a_{U 2} b_{k, U 2} & \ldots & a_{U U} b_{k, U U}\end{array}\right)(t)$

where the time dependence of the $(i, j)$ th element is indicated at the right of the matrix for simplicity. In (2) $a_{i j}$ represents the sign of the element at the $i$ th row and the $j$ th column, which is determined by the STS design rule, while $b_{k, i j}$ is the data bit assigned to the $(i, j)$ th element, which is one of the $U$ input data bits $\left\{b_{k 1}, b_{k 1}, \ldots, b_{k U}\right\}$ of user $k$. Each input data bit of $\left\{b_{k 1}, b_{k 2}, \ldots, b_{k U}\right\}$ appears only once in any given row and in any given column.

\subsection{Channel Model}

The complex low-pass equivalent representation of the impulse response experienced by the $u$ th parallel sub-signal of user $k$ is given by [6]

$$
h_{k}^{u}(t)=\sum_{l=1}^{L} h_{k l}^{u} \delta\left(t-\tau_{k l}\right) \exp \left(j \phi_{k l}^{u}\right),
$$

where $h_{k l}^{u}, \tau_{k l}$ and $\psi_{k l}^{u}$ represent the attenuation factor, delay and phase-shift of the $l$ th multipath component of the channel, respectively, while $L$ is the total number of resolvable multipath components and $\delta(t)$ is the Kronecker Deita-function. We assume that the phases $\left\{\psi_{k l}^{u}\right\}$ in (3) are independent identically distributed (iid) random variables uniformly distributed in the interval $[0,2 \pi)$, while the $L$ multipath attenuations $\left\{h_{k l}^{u}\right\}$ in (3) are independent Nakagami random variables having a Probability Density Function (PDF) of [4]

$$
\begin{aligned}
p\left(h_{k l}^{u}\right) & =M\left(h_{k l}^{u}, m_{k l}^{(u)}, \Omega_{k l}^{u}\right), \\
M(R, m, \Omega) & =\frac{2 m^{m} R^{2 m-1}}{\Gamma(m) \Omega^{m}} e^{(-m / \Omega) R^{2}},
\end{aligned}
$$

where $\Gamma(\cdot)$ represents the gamma function [6], and $m_{k l}^{(u)}$ is the $\mathrm{Na}$ kagarni- $m$ fading parameter, which characterizes the severity of the fading over the $l$-th resolvable path between the $u$ th transmission antenna and user $k$. The parameter $\Omega_{k l}^{u}$ in (4) is the second moment of $h_{k l}^{u}$, i.e., we have $\Omega_{k l}^{u}=E\left[\left(\alpha_{k l}^{u}\right)^{2}\right]$. We assume a negative exponentially decaying multipath intensity profile (MIP) given by $\Omega_{k l}^{u}=\Omega_{k 1}^{u} e^{-\eta(l-1)}, \quad \eta \geq 0$, where $\Omega_{k 1}^{u}$ is the average signal strength corresponding to the first resolvable path and $\eta$ is the rate of average power decay.

We support $K$ asynchronous CDMA users in the system and assume perfect power control. Consequently, when the $K$ users' signals obeying the form of (1) are transmitted over the frequencyselective fading channels characterized by (3), the received complex low-pass equivalent signal at a given mobile station can be expressed as

$$
\begin{gathered}
R(t)=\sum_{k=1}^{K} \sum_{l=1}^{L} \sqrt{\frac{2 P}{U^{2}}} \mathrm{c}\left(t-\tau_{k l}\right) \mathrm{B}_{U}\left(t-\tau_{k l}\right) \mathrm{h}_{k l} \\
\times \operatorname{PN}_{k}\left(t-\tau_{k l}\right)+N(t)
\end{gathered}
$$


where $N(t)$ is the complex valued low-pass-equivalent Additive White Gaussian Noise (AWGN) having a double-sided spectral density of $N_{0}$, while

$$
\mathbf{h}_{k l}=\left(\begin{array}{c}
h_{k l}^{1} \exp \left(j \psi_{k l}^{1}\right) \\
h_{k l}^{2} \exp \left(j \psi_{k l}^{2}\right) \\
\cdots \\
h_{k l}^{U} \exp \left(j \psi_{k l}^{U}\right)
\end{array}\right), \quad \begin{gathered}
k=1,2, \ldots, K \\
l=1,2, \ldots, L
\end{gathered}
$$

represents the channel's complex impulse response in the context of the $k$ th user and the $l$ th resolvable path, where $\psi_{k l}^{u}=\phi_{k l}^{u}-2 \pi f_{c} \tau_{k l}$.

\subsection{Receiver Model}

Let the first user be the user-of-interest and consider a receiver using space-time de-spreading as well as diversity combining, as shown in Fig.1(b), where the subscript of the reference user's signal has been omitted for notational convenience. The receiver of Fig.1(b) carries out the inverse operations of those seen in Fig.1(a), in addition to multipath diversity combining. In Fig.l(b) the received signal is first down-converted using the carrier frequency $f_{c}$, and then descrambled using the DS scrambling sequence of $\operatorname{PN}(t-n)$ in the context of the $l$ th resolvable path, where we assumed that the receiver is capable of achieving near-perfect multipath-delay estimation for the reference user. The de-scrambled signal associated with the $l$ th resolvable path is space-time de-spread using the approach of [2], in order to obtain $U$ separate variables, $\left\{Z_{l l}, Z_{2 l}, \ldots, Z_{U l}\right\}$, corresponding to the $U$ parallel data bits $\left\{b_{1}, b_{2}, \ldots, b_{U}\right\}$, respectively. Following space-time de-spreading, a decision variable is formed for each parallel transmitted data bit of $\left\{b_{1}, b_{2}, \ldots, b_{U}\right\}$ by combining the corresponding variables associated with the $L$ number of resolvable paths, which can be expressed as

$$
Z_{u}=\sum_{l=1}^{L} Z_{u l}, u=1,2, \ldots, U .
$$

Finally, the $U$ number of transmitted data bits $\left\{b_{1}, b_{2}, \ldots, b_{U}\right\}$ can be decided based on the decision variables $\left\{Z_{u}\right\}_{u=1}^{U}$ using the conventional decision rule of a BPSK scheme.

It was shown in [1] that, with the aid of the Gaussian approximation, the average BER of the STS-assisted W-CDMA system using $U$ transmission antennas can be expressed as

$$
P_{b}(E)=\frac{1}{\pi} \int_{0}^{\pi / 2} \prod_{l=1}^{L} \prod_{u=1}^{U}\left(\frac{m_{l}^{(u)} \sin ^{2} \theta}{\bar{\gamma}_{l u}+m_{l}^{(u)} \sin ^{2} \theta}\right)^{m_{l}^{(u)}} d \theta,
$$

where

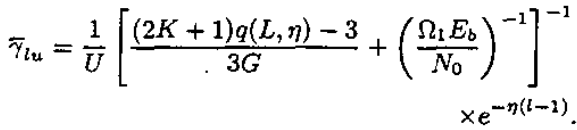

Equation (8) suggests that the diversity order achieved is $L U$ and the diversity gain due to STS appears to be independent of the diversity gain contributed by the RAKE receiver.

In Fig. 2 we compare the BER performance of the STS-assisted W-CDMA system transmitting over flat Rayleigh fading channels and that of the conventional RAKE receiver using only one transmission antenna, but communicating over frequency-selective Rayleigh

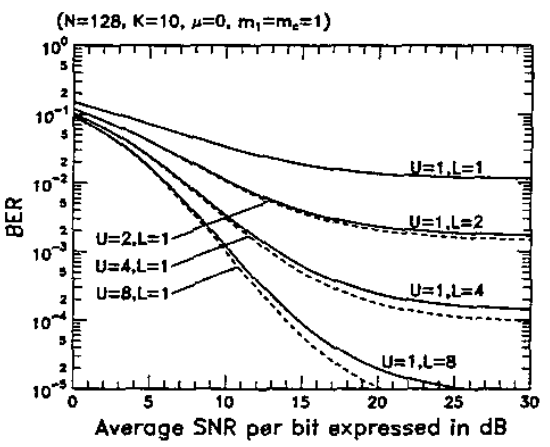

Figure 2: BER versus the SNR per bit, $E_{b} / N_{0}$, performance comparison between the space-time spreading based transmit diversity scheme and the conventional RAKE receiver arrangement using only one transmission antenna when communicating over flat-fading (for space-time spreading) and multipath ( for RAKE) Rayleigh fading ( $m_{l}=m_{c}=1$ ) channels by assuming that the average power decay rate was $\eta=0$.

fading channels. The results in this figure were evaluated from (8) by assuming appropriate parameters, as shown in the figure. Based on those results we observe that both the STS based transmit diversity scheme transmitting over the frequency non-selective Rayleigh fading channel and the conventional RAKE receiver scheme communicating over frequency-selective Rayleigh fading channels having the same number of resolvable paths as the number of transmission antennas in the STS-assisted scheme achieved a similar BER performance, with the STS scheme slightly outperforming the conventional RAKE scheme.

\section{ADAPTIVE SPACE-TIME SPREADING}

The main philosophy behind the proposed ASTS scheme is the realtime adaptive control of the STS based transmission scheme, in order that the system achieves its maximum throughput, while maintaining the required BER performance. In the context of the STS-assisted W-CDMA system, the delay-spread of the wireless channels, and hence the number of resolvable paths available varies slowly over a range spanning from one to dozens of paths. The STS scheme designed based on a low number of resolvable paths, or even based on a relatively high but constant number of resolvable paths, cannot maximize the achievable throughput. A high-efficiency STS based communication scheme must be capable of combining the transmitted energy, which was scattered over an arbitrary number of resolvable paths, and the mode of operation of the STS scheme can be adaptively controlled according to the receiver's detection performance.

The principle of implementing channel-dispersion controlled adaptive rate transmission using ASTS may be readily interpreted by referring to the following example. Let the transmitter employ a total of four transmission antennas. If the number of resolvable paths experienced by the receiver is low, the transmitter is instructed by 
the receiver with the aid of the system's control channel to employ a STS scheme based on four transmit antennas, using the STS scheme described as [2]

$$
\mathbf{S}=\left[\begin{array}{llll}
c_{1} & c_{2} & c_{3} & c_{4}
\end{array}\right]\left(\begin{array}{cccc}
b_{1} & b_{2} & b_{3} & b_{4} \\
b_{2} & -b_{1} & b_{4} & -b_{3} \\
b_{3} & -b_{4} & -b_{1} & b_{2} \\
b_{4} & b_{3} & -b_{2} & -b_{1}
\end{array}\right)
$$

where $c_{1}, c_{2}, c_{3}, c_{4}$ are four STS-related orthogonal codes having a period of $4 T_{b}$. The above STS scheme transmits $U=4$ parallel data bits during the interval of $4 T_{b}$, and hence the effective transmission bit rate becomes $R_{b}=4 \times 1 / 4 T_{b}=1 / T_{b}$. By contrast, when the number of resolvable paths increases, the transmitter is instructed by the receiver to employ four separate STS schemes, each based on two transmit antennas, which can be formulated as:

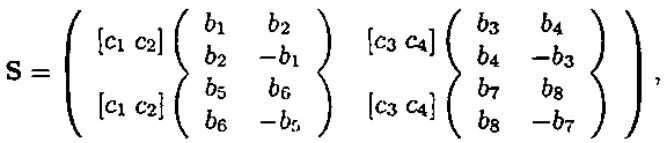

which, again, constitutes four independent 2 -antenna based STS schemes denoted by $\mathbf{B}_{2}(t)$ according to (2), where $c_{1}, c_{2}, c_{3}, c_{4}$ are the $U=4$ STS-related orthogonal codes having a period of $2 T_{b}$. Based on the above four 2 -anternn assisted STS schemes, $U=4$ paralle] data bits are transmitted dunng the first $2 T_{b}$-duration interval using the STS scheme $B_{2}(t)$ of $(2)$ Specifically, antennas 1 and 2 are activated with the aid of $\mathrm{c}$. $\%$. while activating antennas 3 and 4 , when using $c_{3}, c_{4}$. During the following $2 T_{b}$-duration slot another four data bits are transmitted usıng the same scheme as outlined above. Consequently, the above four 2 -antenna based STS schemes transmit a total of eight data bits dunng tuo consecutive $2 T_{b}$-duration timeslots having a total duration of $4 T_{0}$. and the effective transmission rate is now doubled to $2 h_{b}$ Furthermore, if the number of resolvable paths is sufficiently high. which results in requiring no transmit diversity at all. then the four transmission antennas can transmit their information independently, and the corresponding transmission mode can be described as

$$
\mathbf{S}=\left(\begin{array}{cccc}
c_{1} b_{1} & c_{2} b_{2} & c_{3} b_{3} & c_{4} b_{4} \\
c_{1} b_{3} & c_{2} b_{4} & c_{3} b_{3} & c_{4} b_{8} \\
c_{1} b_{0} & c_{2} b_{10} & c_{3} b_{11} & c_{4} b_{12} \\
c_{1} b_{13} & c_{3} b_{14} & c_{3} b_{13} & c_{4} b_{16}
\end{array}\right)
$$

which implies that each of the 16 bis is transmitted independently using an antenna within a dursuon $T_{b}$. where $c_{1}, c_{2}, c_{3}, c_{4}$ are four orthogonal codes having a penod of $T_{b}$. each mapped to one antenna. Explicitly, this scheme is capable of transmitting a total of 16 data bits during an interval of $4 T_{b}$, and hence we achieve a transmission rate of $4 R_{k}$.

The PDF of the delay spread in a wireless communication channel can be approximated by a negative exponential distribution [5] More explicitly, it can be shown that the PDF of the number of resolvabie paths can be expressed as

$$
f\left(\tau_{r}\right)=\frac{1}{T_{m} / T_{c}} \exp \left(-\frac{\tau_{r}}{T_{m} / T_{c}}\right), \tau_{r} \geq 0,
$$

where $T_{m}$ represents the mean square of the distribution, which is also the average value of the delay spread. Some typical examples of $T_{m}$ in different environments are [5]: $T_{m}<0.1 \mu \mathrm{s}$ for an indoor environment, $T_{m}<0.2 \mu s$ for an open rural area, $T_{m} \approx 0.5 \mu \mathrm{s}$ for a suburban area and $T_{m} \approx 3 \mu \mathrm{s}$ for a typical urban area. In (13) $T_{m} / T_{c}$ represents the average delay-spread to chip-duration ratio, and $\left\lfloor T_{m} / T_{c}\right\rfloor+1$ - where $\lfloor x\rfloor$ represents the largest integer not exceeding $x$ - is the average number of resolvable paths, which has been widely used in the performance analysis of DS-CDMA systems transmitting over multipath fading channels.

Let the number of resolvable paths associated with the reference signal be $L_{r}$. For DS-CDMA signals having a chip-duration of $T_{\mathrm{c}}$, the number of near-instantaneous resolvable paths $L_{r}$ can be modeled as a discrete random variable, which varies slowly depending on the communication environment encountered. For a given BER, let the maximum throughput conditioned on the number of resolvable paths $L_{r}$ be $B\left(L_{r}\right)$. Ideally, assuming that the receiver is capable of combining an arbitrary number of resolvable paths and that the transmitter has the perfect knowledge of the number of resolvable path with the aid of a feedback channel, and that the feedback delay is negligible, the unconditional throughput, $B$, using adaptive STS can be written as

$$
B=\sum_{L_{r}=1}^{\infty} P\left(L_{r}\right) \cdot B\left(L_{r}\right),
$$

where $P\left(L_{r}\right)$ is the probability that there are $L_{r}$ resolvable paths at the receiver. With the aid of (13), this probability can be approximated as

$$
\begin{aligned}
P\left(L_{r}\right)= & \int_{\max \left\{0, L_{r}-1-0.5\right\}}^{L_{r}-1+0.5} f\left(T_{r}\right) d \tau_{r} \\
= & \exp \left(-\frac{\max \left\{0, L_{r}-1-0.5\right\}}{T_{m} / T_{c}}\right) \\
& -\exp \left(-\frac{L_{r}-1+0.5}{T_{m} / T_{c}}\right),
\end{aligned}
$$

where $\left[L_{r}-1-0.5, L_{r}-1+0.5\right]$ is the normalized delay-spread range having $L_{r}$ resolvable paths. In (14) $B\left(L_{r}\right)$ represents the maximum possible throughput conditioned on having $L_{r}$ number of resolvable paths. For example, for the proposed ASTS scheme using four-antenna based STS, two-antenna based STS as well as conventional single antenna based transmission, as characterized in (10), (11) and (12), $B\left(L_{r}\right)$ may achieve values of $R_{b}, 2 R_{b}$ or $4 R_{b}$, respectively, depending on the specific number of resolvable paths encountered.

\section{ASTS PERFORMANCE RESULTS}

Figs. 3 and 4 show the throughput versus SNR/bit performance of the ASTS-assisted W-CDMA system using a maximum of four antennas. Depending on the number of resolvable paths at the receiver and on the corresponding achievable BER performance, the transmitter may activate one of the transmission schemes described by (10), (11) and (12). In our related investigations, the target BER was set to 0.01 . Specifically, if a sufficiently high number of resolvable paths is encountered by the receiver, which results in a BER of less than 0.01 for the scheme described by (12), then the transmitter supports a bit-rate of $4 R_{b}$. If the number of resolvable paths is in a range, where the BER using the scheme described by (12) is higher than 0.01 , but that of the STS scheme described by (11) is lower than 0.01 , then the transmitter transmits at a rate of $2 R_{b}$. Finally, if the 


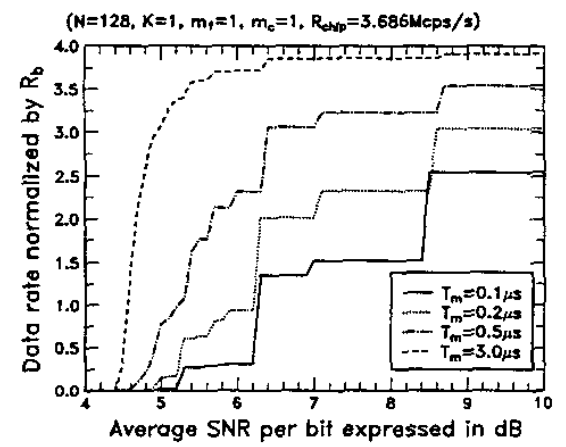

Figure 3: Normalized throughput versus the SNR per bit, $E_{b} / N_{0}$, performance of the adaptive space-time spreading assisted WCDMA system using 4-antenna based STS of (10), the 2-antenna aided STS of (11) and the conventional single-antenna scheme for transmission over four typical wireless channels experiencing Rayleigh fading ( $m=1$ ). The target BER of the reference user is 0.01 and there are no interference users, i.e., $K=1$.

number of resolvable paths is in a range, where the BER using the STS scheme described by (11) is higher than 0.01 , but that described by (10) is lower than 0.01 , then the transmitter transmits at a rate of $R_{b}$. Otherwise, if the number of resolvable paths is too low, which results in BER $>0.01$ for the STS scheme described by (10), then the transmitter simply disables transmissions.

From the resuits of Figs. 3 and 4 we observe that with the aid of the ASTS scheme the system's effective throughput is significantly increased, provided that the average delay-spread of the channel is sufficiently high. Let us highlight the significance of this observation in more detail. Using $T_{m}=0.5 \mu \mathrm{s}$ and $3 \mu \mathrm{s}$ as examples and by observing Fig. 3 we find that the SNR/bit required for transmitting at the data rate of $R_{b}$ is about $5.2 \mathrm{~dB}$ for $T_{m}=0.5 \mu \mathrm{s}$ and 4.6dB for $T_{m}=3 \mu \mathrm{s}$. Similarly, the SNR/bit required for supporting the data rate of $3 R_{\mathrm{b}}$ is about $6.4 \mathrm{~dB}$ for $T_{m}=0.5 \mu \mathrm{s}$ and $5 \mathrm{~dB}$ for $T_{m}=3 \mu \mathrm{s}$. Hence, the adaptive STS-assisted W-CDMA system increased the achievable transmission rate by a factor of three, while requiring only a modest transmitted power increase of about $1.2 \mathrm{~dB}$ for $T_{m}=0.5 \mu \mathrm{s}$ and $0.4 \mathrm{~dB}$ for $T_{m}=3 \mu \mathrm{s}$. Similar results can also be observed in Fig.4, where an extra $0.4 \mathrm{~dB}$ or $1.2 \mathrm{~dB}$ transmitted power is required for achieving a data rate of $3 R_{b}$ instead of $R_{b}$. However, if the number of resolvable paths varies over a relatively low range, the required increase of the transmitted power becomes higher. For example, for the case of $T_{m}=0.1 \mu \mathrm{s}$ in Fig.3 and Fig.4 an extra 2.2dB (Fig.3) or 1.2dB (Fig.4) transmitted power must invested, in order to achieve a data rate of $2 R_{b}$ instead of $R_{b}$. In this scenario, due to the associated extra complexity of the ASTSassisted scheme required by the channel dispersion estimation and feedback, and due to the control channel requirement of the dispersion feedback, the ASTS-aided scheme might not constitute a more attractive alternative.

In the context of the 3rd-generation (3G) W-CDMA systems,

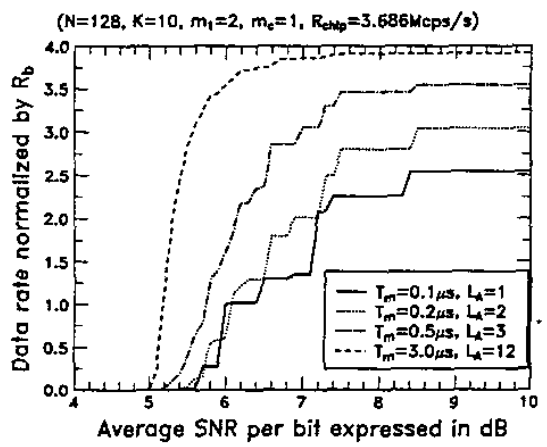

Figure 4: Normalized throughput versus the SNR per bit, $E_{b} / N_{0}$, performance of the adaptive space-time spreading assisted WCDMA system using the 4-antenna based STS of (10), the 2 -antenna aided STS of (11) and the conventional single-antenna scheme for transmission over four typical wireless channels obeying the Nakagami- $m$ distribution $\left(m_{1}=2, m_{c}=1\right)$. The target BER of the reference user is 0.01 , while the interfering users communicate using the 4-antenna based STS of (10) and each interfering signal has an average of $L_{A}$ number of resolvable paths.

since a chip rate of $R_{c} \geq 3.686 \mathrm{Mcps} / \mathrm{s}$ has been employed, the number of resolvable paths may vary over a wide range in urban and suburban areas. Hence, using adaptive STS-aided transmissions constitutes a promising option, when supporting high data rate services.

\section{REFERENCES}

[1] V. Tarokh, N. Seshadri, and A. R. Calderbank, "Space-time codes for high data rate wireless communication: performance criterion and code construction," IEEE Transactions on Information Theory, vol. 44, pp. 744-765, March 1998.

[2] B. Hochwald, T. L. Marzetta, and C. B. Papadias, "A transmitter diversity scheme for wideband CDMA systems based on space-time spreading," IEEE Journal on Selected Areas in Communications, vol. 19, pp. 48-60, January 2001.

[3] L-L. Yang and $L$. Hanzo, "Performance analysis of space-time spreading assisted wideband CDMA systems communicating over multipath Nakagami fading channels," Submitted for Publication, 2001.

[4] T. Eng and L. B. Milstein, "Coherent DS-CDMA performance in Nakagami multipath fading," IEEE Transactions on Communications, vol. 43, pp. 1134-1143, Feb./Mar./Apr. 1995.

[5] W. C. Y. Lee, Mobile Communications Engineering. New York: McGraw-Hill, 2nd ed., 1998.

[6] J. G. Proakis, Digital Communications. McGraw Hill, 3rd ed., 1995. 